\title{
Decomposition of dynamic tasks of the optimum control
}

\author{
Khu Ven-Tsen
}

The method of decomposition for complex multivariate problems of management, based on the account of situations during the moment of acceptance of operating decisions is offered. Unlike known methods of decomposition, it does not show special require- ments to structure of a problem of management. Assumes data of an initial problem of management to the modified problem considering situations, and a problem of identification of the situations, solved in common at two levels.
Optimizational tasks, decisive in automatic systems of control are generally dynamic and must be formulated as follows:

$$
\begin{aligned}
& F(x, u, y, t) \rightarrow \max _{u(t)} \\
& \tilde{o}(t) \in X, u(t) \in U, y(t) \in Y
\end{aligned}
$$

where $x(t)$ - is a vector of revolting influence to the object of control; $u(t)$ - is a vector of control; $y(t)$-is a vector of exits of the object, $t$ - is a variable of time, $f$ - is a criterion function; $x$-set of possible meanings of a variable $x(t)$ to the account; $u, y$-set of the allowable meanings for $u(t)$ and $y(t)$ included.

The function of time may be defined as a decision of the given task that provides maximum criterion function $F(x, y, t)$ in changing in the time of revolting influence.

Generally we can see the task as follows:

$$
\begin{aligned}
& G(x, u, y, t) \rightarrow \max _{u(t) \in U} \\
& U=\{u: g(x, u, y, t)=0 ; h(x, u, y, t) \geq 0\}
\end{aligned}
$$

where $g$ and $h$-are given vector-marked function in a mathematic model of the object of control and restrictions of the task.

All ratios considered in the task as models and restrictions are set in the class of integrate-differential equations and inequalities.

To the industrial object of a scale of complete production, groups of production considered in the given work as the objects of control of a class of complex tech- nological system, the number of similar ratios are usually great, and vectors of variable have a big dimension. It causes the increased complexity of the task of control the CTS, decision of which in an automatic system of control with saving exploitation requirements as a rule is quite complicated or impossible.

In similar situations the use of methods of decomposition can be a decision of the problem, realized in centralized systems of control with hierarchical structural organization.

They suppose to reduce the item of information of an initial task to the set of more simple local tasks by separate structural elements in the structure of the CTS and the task of coordination.

Thus, as a decision of the task of coordination appears such meanings of its variable in which decisions of the local task cause the decision of the initial task of control. In case of its increased complexity we can use the two-leveled procedure of decisions for the local tasks of control. In result a common procedure of the decision of the initial task of control becomes multi-leveled in which the tasks of different levels are connected with hierarchical system of co-subordination.

The methods of decomposition are developed just for statistic tasks of control, but they cannot be used to the dynamic tasks. There is also a decompositional approach to the decision of dynamic tasks of control based on the reduction of the initial task to the consequence of the statistic tasks reflecting current situation on the object of control. In result the decisive task is decomposed by situations.

Thus a simplified statistic task of control may correspond to the considered situation. 
The meaning of the suggested approach is concluded in the following situation;

Let us suppose that the revolting influence $\mathrm{x}(\mathrm{t})$ on the set of (x) have a nature of peaceable-permanent function. Such allowance is quite legal because the basic influences in controlling the objects of the considered class are appeared by changing the parameters of a quality of the processed raw material when there is a transition from one party of the raw material to the next, or changes of loading to technological aggregates.

In both cases a meaning of the revolting parameters is saved without changes for a sufficient long time.

Taking into account this assumption we can see the possible reduction of dynamic task of control to the consequent decisive statistic tasks in the following situation.

$$
\begin{gathered}
F(x, u, y) \rightarrow \max _{u \in U} \\
U=\left\{u: \begin{array}{l}
g(x, u, y)=0 \\
h(x, u, y) \geq 0
\end{array}\right\}
\end{gathered}
$$

Generally the structure of the given task is left unchanged. But if it is still complex and difficult to solve owing to its big dimension, complexity and the large numbers of functional links between the variables, then we can use the methods of decomposition for static tasks of control. The suggested approach is based on that the task of control in the moment of taking controlling decisions is modified by the structure of variables, and criterion function used by mathematic models and considered restrictions. The aim of such modification is to simplify the task to the maximum on the account of reducing the number of variables and corresponding reformations of the criterion function and considered conditions to the minimum.

Thus, the modified task saves its equivalency to the initial task. The result of this modification is a possibility of efficient decision of reformed task with the use of traditional methods of non-linear programming in the frame of centralized systems of control which have not any special demands to the productivity of the calculating devices. Accordingly, taking of controlling decision is realized by solving the tasks of control of the variable structure.

So we can speak about its original decomposition as the initial task of control is reduced to the set of simplified private task which are solved separately and their coordination corresponds to the choice of the private tasks. In this case the decomposition may be tractate as temporary, that is realized in the time but not in the space. However, it does not mean a direct account in the task of factor of time.

In the suggested situation it reflects just the next modification of a task in a moment of taking controlling decisions. It is necessary to note that pointed modification of the initial task of control and its transformation to a more simplified private task certainly means their some rudeness.

Generally we cannot guarantee a complete coincidence of decisions of the initial and modified tasks. It is possible only with some error. So speaking about the equivalency of the given tasks we must take into account that it is implied in definite limits and might be estimated by different parameters of correspondence. However, this circumstance cannot be an obstruction to the practical use of suggested approach. In the ranked decision of the similar tasks in the system of control we cannot define the required optimum, but its some approximation, in the form of any point, from given vicinity of a point of the optimum, that is the decision is always found with some error.

So some disconnection in solving an initial and modificated task is quite allowable. The important thing is that it ought not to exceed its given limiting meaning.

Achieving decisions of the tasks of control with high accuracy is not important in real conditions because their reproduction on the object of control with the use of automatic system of regulation and technical executive devices are always realized with some error, materially exceeding the allowed errors in the decision of the tasks of control.

On the base of the modifications of the tasks of control lies an estimation of the situation in the CTS to the moment of taking controlling decision. Generally under the situation we mean some generalized characteristic of the current condition of the object of control. It can be defined by the set of meanings of coordinates of a condition, structure of its elements, receiving an increment, the size and mark of the given increment, the degree of conformity of settlement meanings of parameters of a condition of object and the degree of the observance of working restrictions.

There are different possible ways of an estimation of the situation in the certain tasks of control. In most cases when adoption of the controlling decision is connected not only with the appearance of the revolting influence on the object, as an estimation of the situation we can use the current meaning of the entrance 
variable $\mathrm{x}$. The structure of an efficient variable of the tasks of control, mathematic models of the CTS and conditions in restrictions are defined in accordance with the suggested approach and on the basis of analysis of the current situation.

In result all insignificant variables are cut out, so the criterion function, the models and restrictions are aptly transited and simplified that lead to the simplification of the tasks of control on the whole.

The important advantage of the suggested approach is that it does not have any special requirements on the structure of the tasks of control, its criterion function and considering conditions as it happened in the methods of decomposition above. The basic requirement here is an opportunity of division and recognition.

Let us look at the substantial party of the suggested approach called decomposition on situations or situate decomposition. The initial task of control is reduced to the set of private subtasks, each of that corresponds to the definite situation in the object of control. There is also an additional task of recognition of the current situation appeared on the object of control, and on account of which there is an appeal to the private subtask. The task of recognition of the situation is tractate as the task of coordination when the private subtask is considered as a counterpart of the local task of control.

The task of recognition of the current situation, identified with a task of coordination is formulated as follows:

$$
\begin{aligned}
& \begin{array}{llll}
0 & 0 & 0 & R
\end{array} \\
& (x, u, y): \rightarrow i, M_{i} \\
& \begin{array}{lll}
0 & 0 & 0
\end{array}
\end{aligned}
$$

where $(x, u, y)$ - are concrete meanings of the entrance variable $\mathrm{x}$, of the control $\mathrm{u}$ and exits of $\mathrm{y}$ objects of control, i - is a number of situation, $\mathrm{Mi}$ - is a set of variable of the task of control, considering in i-situations, $M_{i} \subset M=X \cup U \cup Y, \mathrm{X}, \mathrm{U}, \mathrm{Y}$ - set of variables $\mathrm{x}$, $\mathrm{u}, \mathrm{y}$, as well, R-is an operator of representation of the vector $(\mathrm{x}, \mathrm{u}, \mathrm{y}$, ) in pare with $\mathrm{i}, \mathrm{Mi}$.

The meaning of the formulated task is that vector of the current meanings of the variable $x, u, y$ in a moment of taking decisions are displayed on means of the operator $R$ in the number of situation $i$ and set of $M_{i}$ variables of the task (3), efficient in a given situation. Meanings of the functions $f, g$ and $h$ cannot be taken into account, as they are defined by meanings of their arguments $x, u$, $y$. Private or local task is formulated as follows:

$$
F_{i}\left(x_{i}, u_{i}, y_{i}\right) \rightarrow \max _{u_{i} \in U_{i} \subset U}
$$

$U_{i}=\left\{u_{i}: \begin{array}{c}g_{i}\left(x_{i}, u_{i}, y_{i}\right)=0 \\ h_{i}\left(x_{i}, u_{i} y_{i}\right) \geq 0\end{array}\right\}$

$\cup U_{i}=U$

$i=1,2, . ., N$

Here $i$ - is a number of current situation, $\mathrm{N}$ - is a number of possible situations, $x_{i}, u_{i}, y_{i}$ - modified vectors of the entrances, control and exits of the object, $F_{i}$ - is modified criterion function, caused by modified vectormarked functions, $g_{i}, h_{i}, U$ - set of the allowable decision of the initial task. The tasks suggest two-leveled plan of adoption of controlling decisions. Corresponding system of control gains a structure given in the picture 1 . CTS $, i=1,2, \ldots, N$ - CTS, modified with $i$-situations, COis a coordinating organ, MS - is a managing system, deciding the modified task of control.

In a suggested systems of control, CO makes current estimation of the situation in CTS by its identification on the set of considered situations and forms a structure of modified task on the basis of a task on account of excluding the variables of a small importance.

Co-component composition of vectors of the variables $x_{i}, u_{i}, y_{i}$ in the task may be different and implies some numerous $M_{i}$, number of full composition variables M. Components of vectors $x_{i}, u_{i}, y_{i}$ having been chosen by this way are most efficient in this situation.

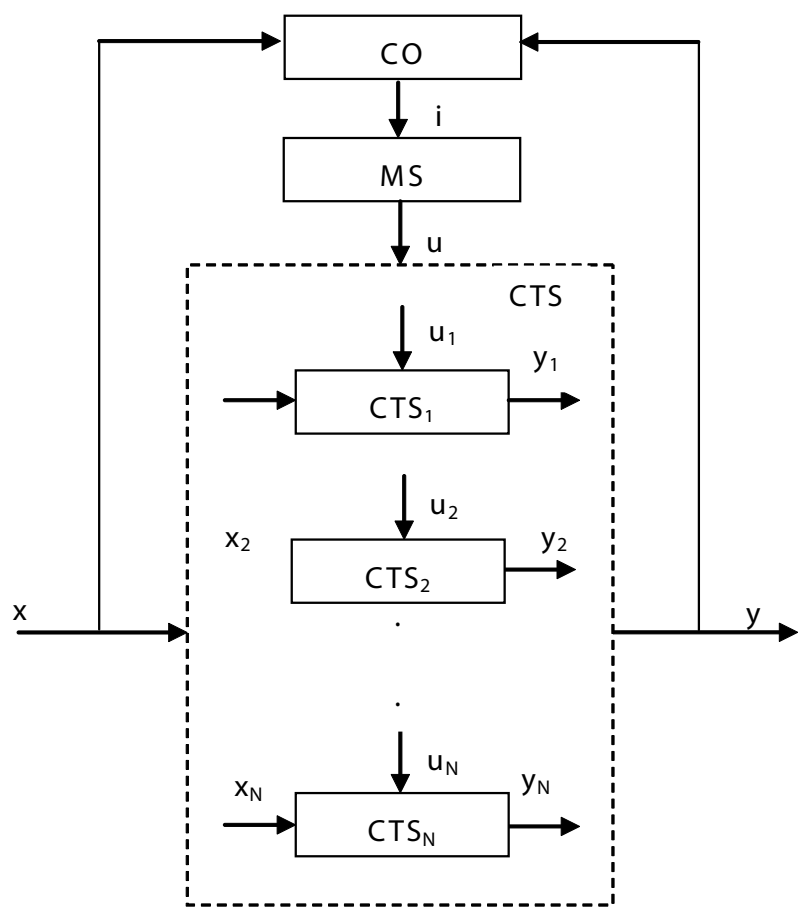

Picture 1: The structure of systems of control, realizing the method of situate decomposition. 
The suggested principle of control may be considered as a counterpart of an adaptive control. The difference in this case is that the identification of situation on the object is realized with consecutive change of the initial task of control of modified task, instead of identification of the mathematic model of the object control.

So in case of adaptive control the structure of a model and tasks of control remain without changes, but the structure of modified task might change.

Generally identification of the situation is connected with the decision of the task of its recognition, the content and methods of decision which depends on specification of the objects of control and considering situation in managing given object.

So the system of control gains the property of a system with a variable structure and the object of control becomes decomposed on situations.

The system of control is centralized and distributed at the same time, connecting the principle of centralized control in the frame of current situation with decomposition of the object of control on situations, causing decentralization of control on the whole.

\section{Literature}

Ven-Tsen, Khu; Volodin, V. M. (2007): About the algorithm of decomposition in the tasks of optimization of chemical-technological system ZH. TOHT AN UCSR, t. HP, No 6, p. 889-895.

Ven-Tsen, Khu; Umbetov, U. (2007): Decentralized control of multimeasured objects with decomposition on situations. ISSN 0002 3191. Intelligence of the National Academy of Science of the Republic of Kazakhstan, the physical- mathematical series, 2007, p.82-85.

Author

Khu Ven-Tsen

South Kazakhstan State University named M. Auezov.

Shymkent city, Kazakhstan 